\title{
Recurrence of tuberculosis in a low-incidence setting
}

\author{
C.C. Dobler*, A.B.H. Crawford*, P.J. Jelfs ${ }^{\#}$, G.L. Gilbert" ${ }^{\#}$ and G.B. Marks*
}

\begin{abstract}
Recurrence of active tuberculosis following treatment of an initial disease episode can occur due to endogenous re-activation or exogenous re-infection.
\end{abstract}

Cases of recurrent tuberculosis in the Australian state of New South Wales between 1994 and 2006 were identified by data linkage analysis with confirmatory review of case notes. Patients with more than one culture-positive disease episode during that time period who had completed treatment for the initial disease episode were included. Genotyping of Mycobacterium tuberculosis was used to determine whether recurrence was likely to be due to re-activation or re-infection.

There were 5,723 tuberculosis notifications between 1994 and 2006, 3,731 of which were culture-positive. Fifteen $(0.4 \%)$ patients had recurrent culture-positive disease over a mean 5.7 yrs of follow-up (crude annual incidence 71 per 100,000 population). Recurrent tuberculosis was attributable to re-activation (indistinguishable strains) in 11 (73\%) cases and to re-infection (different strains) in four (27\%).

In a low-incidence setting of tuberculosis, a control programme incorporating directly observed therapy for active disease resulted in a very low rate of recurrent tuberculosis over a long period of follow-up. Re-infection is less likely than re-activation, but still contributes significantly to the number of cases with recurrent disease.

KEYWORDS: Directly observed therapy, genotyping, re-activation, recurrence, re-infection, tuberculosis epidemiology

$\mathbf{R}$ ates of recurrence of tuberculosis (TB) reflect the long-term efficacy of TB treatment, the effectiveness of the TB control strategy, the underlying incidence of $\mathrm{TB}$ in the community and the immune status of the community. Rates of recurrent TB in settings with a low-to-intermediate incidence of TB are reported to range $1.2-7.0 \%$ [1-4]. Recurrence of active TB following treatment of an initial disease episode may occur due to endogenous re-activation with the same strain of Mycobacterium tuberculosis or to exogenous re-infection with a different strain. In countries with a low or intermediate incidence of $\mathrm{TB}$, recurrence is usually suspected to occur due to re-activation rather than re-infection [1-8]. In contrast, in high-incidence countries, re-infection seems to be a major cause of recurrent disease [9, 10]. HIV infection increases the risk of recurrent disease because HIV-positive individuals infected with $M$. tuberculosis rapidly progress to disease. Furthermore, there is some evidence that they may have a higher risk of developing infection following exposure to $M$. tuberculosis [11]. Traditionally, it was assumed that recovery from an episode of TB at least partially protected an individual against subsequent $\mathrm{TB}$ due to reinfection with another strain. However, a recent study from South Africa has suggested that people who have had TB once are at increased risk of having another episode of disease [9].

In New South Wales (NSW; Australia), the incidence of TB and HIV infection are both low. Of TB cases, $>80 \%$ occur in people born overseas, presumably representing re-activation of latent TB infection. Virtually all patients are treated with directly observed therapy (DOT). The aim of the present study was to establish the incidence of recurrent disease and the relative contributions of re-activation and re-infection in this setting.

\section{PATIENTS AND METHODS \\ Setting}

The present study was conducted in NSW, Australia's most populous state, with a population of $\sim 6,800,000$ (almost a third of Australia's total population). The incidence of TB in NSW is 6.5 per 100,000 population [12]. TB treatment is provided free of charge through local chest clinics. Chest clinics are public-hospital-associated outpatient centres that are specialised in the management of TB.
AFFILIATIONS

*Respiratory Dept, Liverpool

Hospital, and

${ }^{\#}$ Centre for Infectious Diseases and Microbiology, Institute of Clinical Pathology and Medical Research, Westmead Hospital, Sydney, Australia.

CORRESPONDENCE

C.C. Dobler

Liverpool Hospital

Respiratory Dept

Locked bag 710

Liverpool BC NSW 1875

Australia

Fax: 61298294102

E-mail: c.dobler@unsw.edu.au

Received:

July 092008

Accepted after revision:

September 132008

SUPPORT STATEMENT

The National Health and Medical

Research Council Centre of Clinical

Research Excellence in Respiratory

and Sleep Medicine (Sydney,

Australia) provided funding for $\mathrm{C}$.

Dobler. This funding was

independent of this specific study.

STATEMENT OF INTEREST

None declared.

European Respiratory Journal

Print ISSN 0903-1936

Online ISSN 1399-3003 


\section{Study population}

TB is a notifiable disease in NSW. There is evidence that the NSW TB notifications database is a complete, and hence sensitive, record of cases of TB in NSW [13]. All TB notifications between 1994 and 2006 in this database were reviewed. Data linkage within the database was used to identify cases that had experienced more than one TB notification during that time period. Patients were included in the analysis if, between 1994 and 2006, they had had more than one disease episode, both of which were culture-positive, and they had completed treatment for the initial episode of TB.

\section{Data linkage, data analysis}

Records within the NSW TB notification database with an identical date of birth were identified via a computer-generated search. These pairs of records were then manually checked for similar names, sex, country of origin and date of arrival in Australia. Notification dates in different records for the same patient had to be $\geqslant 6$ months apart, otherwise it was assumed that they were duplicate notifications for the same episode, e.g. cases where patients were transferred to another chest clinic during treatment and both institutions notified the patient. Cases with multiple notifications that were $\geqslant 6$ months apart were further analysed by accessing clinical records. Duplicate notifications for the same episode or apparent recurrences with culture-negative disease were excluded. Verification of culture results (by accessing the clinical records) was sought for all cases of recurrence, even if the database stated negative cultures. For the whole cohort of TB notifications, a case was accepted as being culture-positive if either or both of the following were recorded: 1 ) positive culture for $M$. tuberculosis from a dated specimen; and 2) culture was stated to be the disease-identifying method. Cases of multidrug-resistant (MDR) TB were identified from the records of the NSW Mycobacterium Reference Laboratory at the Institute of Clinical Pathology and Medical Research (Westmead Hospital, Sydney, NSW, Australia).

\section{Treatment}

Patients were treated in accordance with the NSW Department of Health (Sydney, Australia) TB guidelines, which require DOT for $\geqslant 6$ months, initially using isoniazid, rifampicin, pyrazinamide and ethambutol for $\geqslant 2$ months, followed by rifampicin and isoniazid for the subsequent 4 months, in fully sensitive disease [14]. All patients were directly observed taking all doses of their medication at the chest clinics or in their home, except that patients on daily treatment were not observed taking their weekend doses. Treatment regimens were no different whether a patient received medication at the chest clinic or at home. Cases of MDR-TB were treated with DOT using second-line treatment over a period of 1224 months.

\section{Follow-up period}

Patients were usually subject to active radiological surveillance for $\geqslant 2$ yrs following treatment cessation. For the present analysis, the mean time from cessation of treatment to December 2006, when the linkage analysis was performed, was 5.7 yrs.

\section{Genotyping of Mycobacterium tuberculosis isolates}

One $M$. tuberculosis isolate from each disease episode from each patient with culture-positive recurrent $\mathrm{TB}$ was used for genotyping. Genotyping was performed at the time of the second disease episode or retrospectively, for the present study, from stored culture material at the Institute of Clinical Pathology and Medical Research. Cross-contamination was excluded by the use of appropriate molecular laboratory techniques, i.e. the use of positive, negative and contamination controls and by testing unrelated isolates in the same batch. DNA fingerprinting was performed by using a combination of three methods, namely spoligotyping [15], mycobacterial interspersed repetitive unit (MIRU) typing [16] and insertion sequence IS6110 restriction fragment length polymorphism (RFLP) typing [17]. Isolates were classified as distinct if they were clearly different by any of these three methods. RFLP typing was usually only performed for isolates that were indistinguishable following spoligotyping and MIRU typing. Disease attributable to re-infection was defined as a recurrent disease episode in which the strain had a genotype distinct from that which was isolated during the initial episode. Recurrences in which the strains from the two episodes were indistinguishable were attributed to re-activation.

\section{Data analysis}

Incidence rates were calculated per person-year of follow-up; 95\% confidence intervals (CIs) around the estimated rates were calculated according to the asymptotic method of FLEISS [18]. The Kaplan-Meier method was used to construct a survival curve, with survival defined as being free of active TB.

\section{Ethical considerations}

Ethical approval was obtained from the New South Wales Population and Health Services Research Ethics Committee (Sydney, Australia).

Preliminary data from the present study (results from one chest clinic in NSW) have been reported previously [19].

\section{RESULTS}

\section{Description of the cohort}

There were 5,723 TB notifications between 1994 and 2006, 3,731 of which were culture-positive (fig. 1). Characteristics of the cohort patients are shown in table 1 . The mean \pm SD age of the study population was $46.7 \pm 21.1 \mathrm{yrs}$, and 1,935 (51.9\%) cases of the culture-positive cohort were male. Information on the country of birth was available for 3,608 of these cases. Of these, $509(14.1 \%)$ were Australian-born. The majority of the overseas-born cases originated from Asia.

\section{Incidence of recurrent tuberculosis}

Fifteen $(0.40 \%$; 95\% CI $0.23-0.68)$ patients had recurrent culture-positive disease following completion of treatment for an initial disease episode over a mean of 5.7 yrs of followup. The recurrent episodes occurred 1.5-100 months (median 17 months) after cessation of treatment for the initial disease episode (fig. 2). The incidence of recurrent culture-positive TB was 70.7 (95\% CI 16.6-241.6) per 100,000 person-yrs of followup. Table 1 shows the characteristics of the TB cohort as a whole and also the cases with recurrent TB. Owing to the low number of cases of recurrent TB, no formal statistical tests were performed. Paired DNA fingerprints were available for all 15 


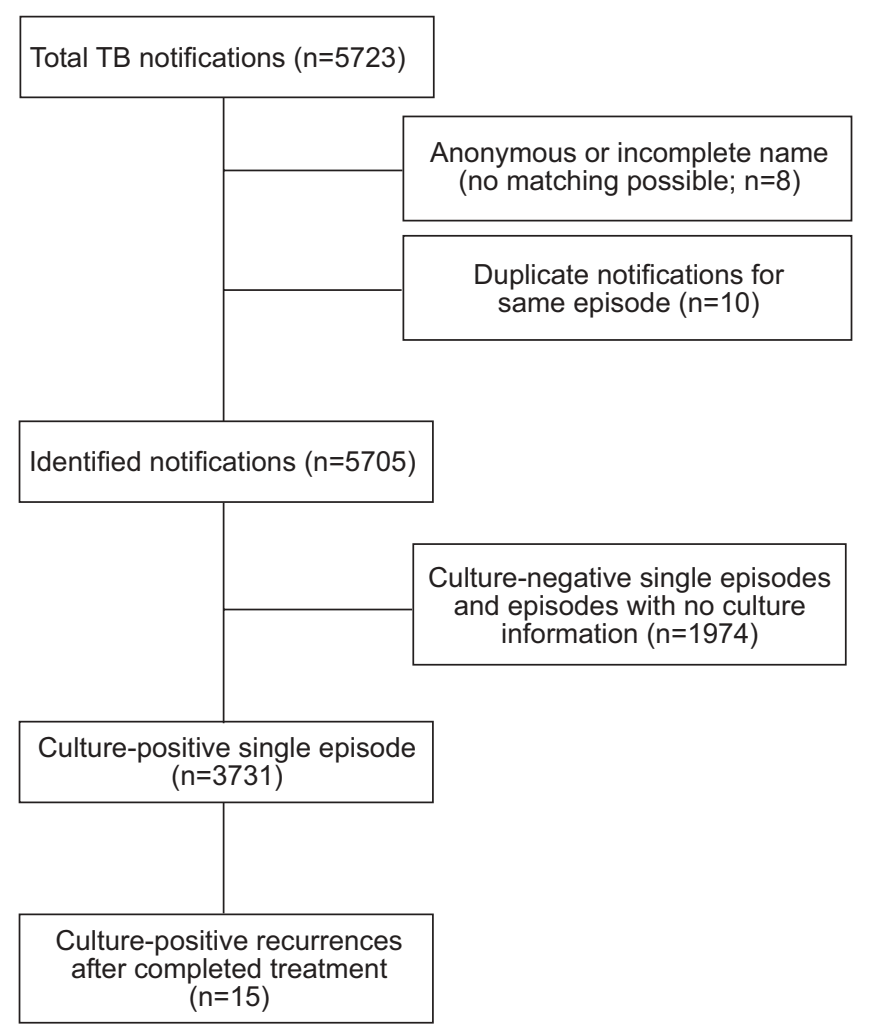

FIGURE 1. Flow chart showing tuberculosis (TB) notifications during the period 1994-2006.

cases. Recurrent TB was attributable to re-activation (indistinguishable strains) in 11 cases and to re-infection (different strains) in four. (Table 2 summarises the clinical and molecular typing data.)

Of the 51 cases with MDR-TB in the initial or only episode, only one had recurrent disease, and that proved to be due to re-infection with a strain that was resistant to isoniazid alone (patient 2; table 2). Of the remaining 14 cases with recurrent disease, three had MDR-TB in the second disease episode, two of which were due to re-activation and one to re-infection with an MDR-TB strain, having initially been infected with a fully sensitive strain.

Among all TB notifications, including culture-negative cases, there were 26 recurrent cases out of 5,723 $(0.45 \%)$ notifications. Hence the estimate of the recurrence rate was not altered by limiting the analysis to culture-positive cases alone.

\section{Re-activation}

Re-activation occurred in 11 (0.29\%; 95\% CI 0.15-0.54) cases. The time to recurrence for re-activation cases ranged 1.5100.0 months (median 21.5 months) after cessation of treatment for the initial disease episode. The annual incidence of reactivation was 51.9 (95\% CI 8.6-213.3) cases per 100,000 personyrs of follow-up. Of the 11 cases with re-activation, two had MDR-TB in the second disease episode. One of these patients (patient 10; table 2) initially had a fully sensitive organism. The patient was diagnosed with HIV/AIDS at the time of presentation with the first episode of active TB. Severe diarrhoea, possibly related to HIV/AIDS, was an ongoing issue in this patient, and might have altered the absorption of the TB medication (no drug level tests were performed). The patient had recurrent disease just 6 weeks after completion of treatment for the first disease episode. In the other case with MDR-TB on re-activation (patient 12; table 2), the initial episode was attributable to an organism that was resistant to isoniazid. The patient was treated with isoniazid, rifampicin, pyrazinamide and ethambutol for 9 months, and presented with re-activated disease 10 months after cessation of treatment for the first episode.

\section{Re-infection}

Re-infection occurred in four $(0.11 \%$; $95 \%$ CI $0.03-0.29)$ out of all culture-positive cases during the period of follow-up. The time to recurrence for re-infection cases ranged 8.0 56.5 months (median 16.3 months) after cessation of treatment for the initial disease episode. The crude annual incidence of recurrent disease due to re-infection was 18.9 (95\% CI $0.3-$ 160.9 ) cases per 100,000 person-yrs, which is four and a half times the incidence of new culture-positive TB (4.2 cases per 100,000 person-yrs).

Of the four cases with recurrent TB due to re-infection, at least three (patients 1-3; table 2 ) had visited countries with a high incidence of TB following treatment of their initial episode in Australia. It was assumed that re-infection occurred during these visits. One of these patients (patient 3; table 2) had received an autologous bone marrow transplant for multiple myeloma and was undergoing treatment with $\alpha$-interferon prior to both episodes of TB. Although this patient was born in Australia, the patient had visited a country with a high incidence of TB between completion of treatment for the first disease episode and the episode of recurrent disease. The number of re-infection cases was too small for statistical analysis of risk factors for exogenous re-infection.

\section{Molecular typing}

The differences found in strains attributed to re-infection were of significance beyond consideration of a mutational event. Only a quarter of the present patient isolates showed an identical spoligotype, but, in five out of 12 of the MIRU loci in these isolates, the repeat numbers differed by 1-2.

The isolates from two patients had been typed by RFLP (the only method available in 1997) at the time of their recurrent disease. In both cases, the paired isolates were indistinguishable, indicating re-activation. These isolates were no longer available for spoligotyping and MIRU typing. There was a range of spoligotype families among the remaining isolates, the commonest of which was Beijing (table 2); two of three MDR isolates for which spoligotyping results were available were Beijing. One patient had separate episodes of infection with two different Beijing strains (patient 2; table 2). In general, spoligotypes reflected the patients' countries of origin.

\section{DISCUSSION}

The present study shows that, in the current population, living in a setting with a low incidence of $\mathrm{TB}$ and low prevalence of $\mathrm{HIV}$ infection and managed with DOT, there was a very low incidence of recurrent TB. The annual incidence of recurrent $\mathrm{TB}$, over a mean period of follow-up of $5.7 \mathrm{yrs}$, was 71 cases per 100,000 person-yrs. Only $0.4 \%$ of all culture-positive 
TABLE 1 Characteristics of tuberculosis (TB) notification cohort and cases of recurrent TB

\begin{tabular}{|c|c|c|c|c|}
\hline & \multirow[t]{2}{*}{ Cohort } & \multicolumn{3}{|c|}{ Cases } \\
\hline & & Recurrent TB & Exogenous re-infection & Endogenous re-activation \\
\hline Subjects $n$ & 3731 & 15 & 4 & 11 \\
\hline Age yrs & $46.7 \pm 21.1$ & $48.5 \pm 22.4^{\#}$ & $31.3 \pm 15.5^{\#}$ & $54.8 \pm 21.6^{\#}$ \\
\hline \multicolumn{5}{|l|}{ Sex } \\
\hline \multicolumn{5}{|l|}{ Country of origin } \\
\hline Data available $n$ & 3608 & 15 & 4 & 11 \\
\hline Australia & $509(14.1)$ & $3(20.0)$ & $1(25.0)$ & $2(18.2)$ \\
\hline Overseas & 3099 (85.9) & $12(80.0)$ & $3(75.0)$ & $9(81.8)$ \\
\hline \multicolumn{5}{|l|}{ HIV status } \\
\hline Assessed $n$ & 507 & 6 & 2 & 4 \\
\hline Positive & $1427(43.8)$ & $10(71.4)^{\#}$ & $4(100)^{\#}$ & $7(70.0)^{\#}$ \\
\hline Negative & $1834(56.2)$ & $4(28.6)^{\#}$ & $0(0)^{\#}$ & $3(30.0)^{\#}$ \\
\hline \multicolumn{5}{|l|}{ Site of disease } \\
\hline Data available $n$ & 3606 & 15 & 4 & 11 \\
\hline Pulmonary only & 2137 (59.3) & $12(80.0)^{\#}$ & $3(75.0)^{\#}$ & $9(81.8)^{\#}$ \\
\hline Pulmonary and extrapulmonary & $257(7.1)$ & $3(20.0)^{\#}$ & $1(25.0)^{\#}$ & $2(18.2)^{\#}$ \\
\hline Extrapulmonary alone & $1212(33.6)$ & $0(0)^{\#}$ & $0(0)^{\#}$ & $0(0)^{\#}$ \\
\hline MDR-TB & $51(1.4)$ & $1(6.7)^{\#}$ & $1(25.0)^{\#}$ & $0(0)^{\#}$ \\
\hline
\end{tabular}

Data are presented as mean \pm sd or $n(\%)$ unless otherwise indicated. MDR: multidrug-resistant. ${ }^{*}$ : at time of first disease episode; ": at least on one specimen of the following: sputum, bronchial washings/lavage, and lymph node fine needle aspiration.

notifications were for recurrent disease. The proportion of cases that recurred in NSW appeared to be substantially lower than that previously reported in comparable studies of treatment outcomes in low-to-intermediate-incidence populations, ranging $1.2-7.0 \%$ [1-4], the majority of which did not utilise DOT.

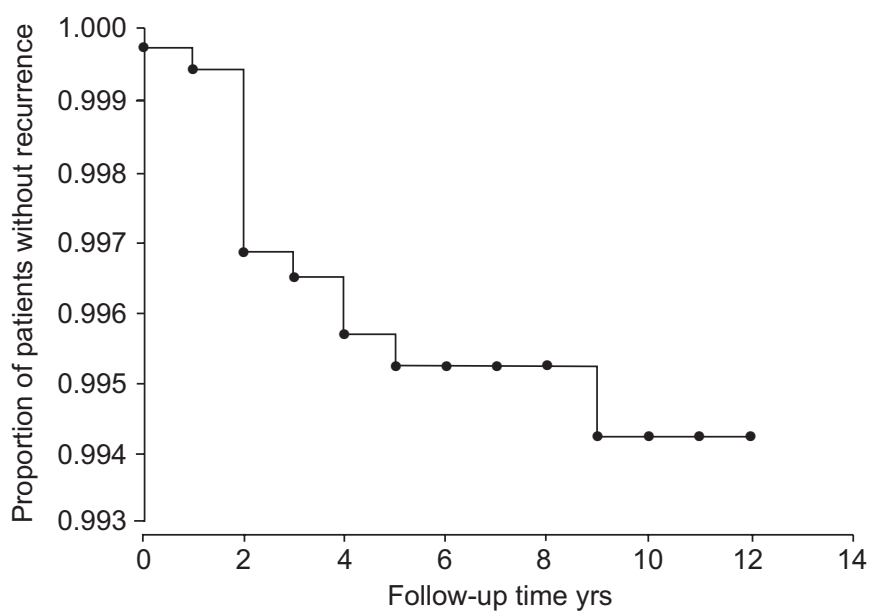

FIGURE 2. Kaplan-Meier curve showing risk of recurrence in 3,731 culturepositive tuberculosis notifications.
Although the number of patients with MDR-TB was small, the present outcomes of DOT with second-line drugs are encouraging. Indeed, none of the patients treated for MDR-TB in a first disease episode showed re-activation disease with the initial organism during follow-up.

Recurrent TB is most likely to occur with drug-susceptible organisms. The present finding that only two out of 11 cases with re-activation had MDR-TB in the second disease episode is consistent with the findings of others $[1,2,8,21,22]$. This finding supports the present authors' current practice of not routinely starting patients on second-line treatment in cases of recurrent TB.

In countries with a low or intermediate incidence of $T B$, recurrent $\mathrm{TB}$ is mainly attributable to re-activation. In this setting, the proportion of recurrent cases attributable to reinfection has been reported to range 4-33\% [1-7]. In contrast, in high-incidence countries, re-infection may account for up to $77 \%$ of cases of recurrent disease $[9,10]$. Although the rate of re-infection reflects the incidence of TB in the area, a low rate of re-activation is a consequence of treatment efficacy, in terms of both optimal drug therapy and patient compliance. The low rate of re-activation in the present study has led to a relatively higher proportion $(27 \%)$ of recurrent cases attributable to re-infection. The relatively high incidence of recurrent disease due to re-infection in the present population, relative to the very low incidence of new-onset culture-positive disease, may also 


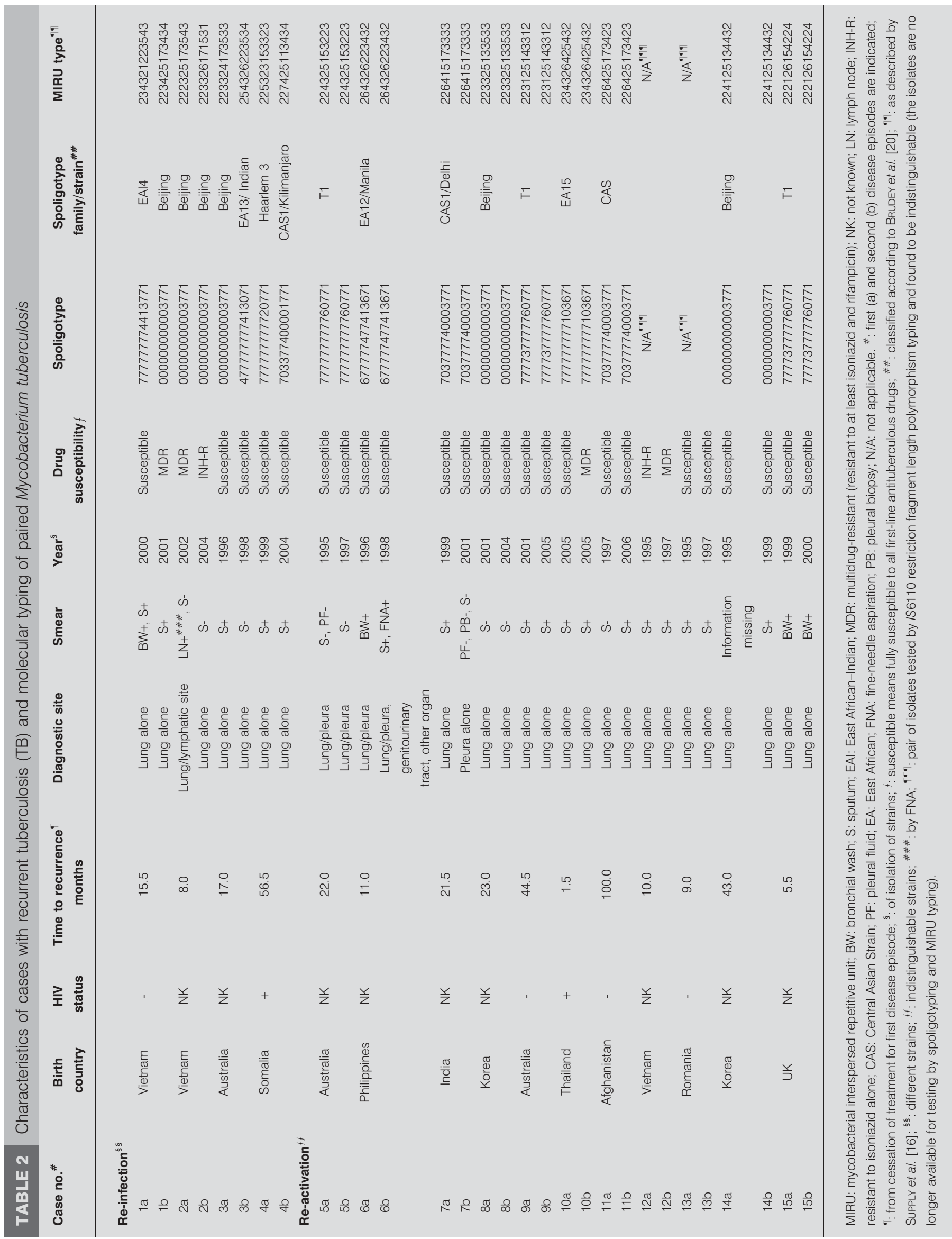


relate to high rates of overseas travel among Australians, particularly those who were born overseas. Travellers to areas of high TB endemicity have been shown to be at substantial risk of TB infection and disease [23, 24]. Of the four cases with reinfection, at least three had had stays in a country with a high incidence of TB following their initial treatment in Australia.

One strength of the present study is the use of data from a TB control programme, which is more representative of real-life practice than experience from clinical trials. However, there are also limitations associated with the use of such data. The cohort was not limited to patients who met the criteria for cure as defined by the World Health Organization [25], but also included patients that were classified as having completed treatment. This is because follow-up culture results were not routinely documented. There were two cases of recurrence within 6 months following completion of treatment for the first episode. One case with re-activation after 6 weeks (patient 10; table 2) showed evidence of sputum smear conversion during treatment, but no sputum examination was performed during the final month of treatment. The case with re-activation after 5.5 months (patient 15; table 2) was initially diagnosed using bronchial lavage specimens rather than sputum, and follow-up bronchoscopy was not routinely performed. Hence the possibility that these two cases represent primary treatment failure rather than recurrent disease cannot be excluded. A further limitation of the present study is that it is not possible to account for recurrent cases that occurred among patients who left NSW during the follow-up period. If a similar rate of departure from NSW as was found in a random sample of 200 refugees who arrived in Sydney, NSW, between 1984 and 1994 [13] is assumed, it is estimated that $15 \%$ of the cohort left NSW during the mean follow-up period of 5.7 yrs. However, the present cohort includes many longer-term residents of NSW and this is likely to be an overestimate of the rate of emigration. Hence this is likely to have only a small impact on the estimates.

The reasons for the low re-activation rate in the present study population can only be the subject of speculation, since the lack of a control group does not permit any definite conclusions to be drawn. One possible explanation is that treatment for TB was administered by units specialised in the management and treatment of TB disease under DOT. The present authors consider that the consistent application of DOT, as strongly advocated by the World Health Organization in treatment programmes for active TB, may be at least partially responsible for the good treatment outcomes. Nevertheless, the present authors are aware that a number of prospective randomised trials in high-incidence populations have failed to demonstrate that DOT, per se, results in improved outcomes in the treatment of active TB $[26,27]$. Another possible explanation for the low re-activation rate may be the concentration of TB treatment in specialised centres, which ensures that standardised treatment regimens of proven efficacy, using the recommended doses for the recommended times, are prescribed. Besides supervision, specialised centres can offer appropriate treatment support, which is especially important in noncompliant patients and patients experiencing possible side-effects of TB treatment. In low-incidence settings, concentration of all TB treatment in specialised centres is also likely to improve the expertise of the health professionals that form part of a treatment team. This may also improve the management of MDR-TB patients. Finally, the fact that, in NSW, all TB treatment is provided free of charge, independent of insurance and residence status, may have improved the compliance of patients with the treatment regimen and hence led to good outcomes.

Although the incidence of re-infection disease (18.9 cases per 100,000 person-yrs) was low, it was still four and a half times that of new culture-positive TB (4.2 cases per 100,000 personyrs). A study from South Africa, a country with a high incidence of TB, has reported a similar finding [9]. However, the incidence of re-infection among foreign-born persons (17.6 cases per 100,000 person-yrs) was similar to the overall incidence of culture-positive TB among foreign-born persons in NSW (16.2 cases per 100,000 person-yrs). This is a more realistic comparison because this is the population within which $85 \%$ of cases of TB occur. Thus, within this population, re-infection occurs at a similar rate to initial infection.

In the present cohort, 507 (13.6\%) out of 3,731 patients had their HIV status assessed, of whom 64 (12.6\%) were HIVpositive. More recently, there have been efforts to introduce routine HIV testing for all TB cases in NSW. In 2005, among $393(37 \%$ of all notified cases that year) cases in which HIV status was assessed, only nine (2.3\%) were HIV-positive. These findings suggest that, in earlier years, when significantly fewer patients were tested for HIV, testing was biased towards patients at higher risk of HIV. Although the numbers of cases with recurrent $\mathrm{TB}$ that were tested for HIV were very small, the present data show that HIV-positive status tends to be more prevalent in patients with recurrent disease than in the whole cohort of TB notifications (table 1).

Recently developed molecular typing methods have made it relatively easy to distinguish re-infection from re-activation, and the PCR-based methods (spoligotyping and MIRU typing) can be performed on nonviable isolates or stored DNA samples. For RFLP typing, the organism must be viable and cultured for a considerable period in order to generate the relatively large amount of bacterial DNA required. Although the combination of three typing methods provides the highest level of discrimination, isolates that are indistinguishable by a combination of spoligotyping and MIRU, in the context of recurrent disease, are almost certain to belong to the same strain. Spoligotyping alone is less discriminatory, especially in populations in which the Beijing family is relatively common. One patient (patient 2; table 2) had two episodes of infection with Beijing family strains that were indistinguishable by spoligotyping but easily distinguished by MIRU typing. The most common genotype in NSW is Beijing (26\%) [28]. Of the 11 cases with re-activation, nine had information on spoligotype family/strain available. Two (22.2\%) were cases with Beijing family strains, which reflects the prevalence of the Beijing genotype in NSW.

There is some uncertainty regarding the classification of cases as attributable to re-infection or re-activation on the basis of strain typing. For example, one of the 11 patients (patient 9; table 2), who had recurrent disease attributable to the same strain as the initial disease episode, was part of a known cluster of TB cases that was confirmed by genotyping and epidemiological linkage. Members of this disease cluster had 
continuing contact with each other at the time the second disease episode occurred [29]. Hence it is possible that, in this case, the second disease episode was due to exogenous reinfection with the same strain rather than re-activation. Conversely, there is also a theoretical possibility that cases classified as re-infection due to different strains had had a simultaneous infection with different strains at the time of the initial episode. If only one genotype was detected initially and the other genotype was detected at the time of recurrence this would have been falsely classified as being attributable to reinfection [30]. Although the likelihood of this is low, this possibility cannot be entirely excluded.

It is concluded that, in an area of low tuberculosis incidence, where virtually all cases of active disease are managed by directly observed therapy in specialised centres, the rate of tuberculosis recurrence is extremely low. Furthermore, disease due to re-activation is more likely than not to be drug-sensitive. In this setting, with high rates of travel to endemic countries, reinfection contributes over a quarter of all cases of recurrence and is likely to be acquired during visits to high-incidence countries. Patients who have been effectively treated for active tuberculosis remain susceptible to re-infection disease, especially when travelling to countries with a high incidence of tuberculosis. Multidrug-resistant tuberculosis is no more likely to relapse than fully sensitive disease, possibly suggesting that treatment through centres that are specialised in the management of tuberculosis and treat every case of active disease with directly observed therapy results in successful control of multidrugresistant tuberculosis in a low-incidence setting.

\section{ACKNOWLEDGEMENTS}

The authors would like to thank the New South Wales Department of Health (Sydney, Australia) for granting access to the tuberculosis notification data for database linkage, and the area tuberculosis coordinators of New South Wales for helping access clinical records.

\section{REFERENCES}

1 Bandera A, Gori A, Catozzi L, et al. Molecular epidemiology study of exogenous reinfection in an area with a low incidence of tuberculosis. J Clin Microbiol 2001; 39: 2213-2218.

2 Cacho J, Pérez Meixeira A, Cano I, et al. Recurrent tuberculosis from 1992 to 2004 in a metropolitan area. Eur Respir J 2007; 30: 333-337.

3 Jasmer RM, Bozeman L, Schwartzman K, et al. Recurrent tuberculosis in the United States and Canada: relapse or reinfection? Am J Respir Crit Care Med 2004; 170: 1360-1366.

4 García de Viedma D, Marín M, Hernangómez S, et al. Tuberculosis recurrences: reinfection plays a role in a population whose clinical/epidemiological characteristics do not favor reinfection. Arch Intern Med 2002; 162: 1873-1879.

5 de Boer AS, Borgdorff MW, Vynnycky E, Sebek MM, van Soolingen D. Exogenous re-infection as a cause of recurrent tuberculosis in a low-incidence area. Int J Tuberc Lung Dis 2003; 7: 145-152.

6 Sudre P, Pfyffer GE, Bodmer T, et al. Molecular epidemiology of tuberculosis among HIV-infected persons in Switzerland: a countrywide 9-year cohort study. Infection 1999; 27: 323-330.
7 El Sahly HM, Wright JA, Soini H, et al. Recurrent tuberculosis in Houston, Texas: a population-based study. Int J Tuberc Lung Dis 2004; 8: 333-340.

8 Caminero JA, Pena MJ, Campos-Herrero MI, et al. Exogenous reinfection with tuberculosis on a European island with a moderate incidence of disease. Am J Respir Crit Care Med 2001; 163: 717-720.

9 Verver S, Warren RM, Beyers N, et al. Rate of reinfection tuberculosis after successful treatment is higher than rate of new tuberculosis. Am J Respir Crit Care Med 2005; 171: 1430-1435.

10 van Rie A, Warren R, Richardson M, et al. Exogenous reinfection as a cause of recurrent tuberculosis after curative treatment. N Engl J Med 1999; 341: 1174-1179.

11 Sonnenberg P, Murray J, Glynn JR, Shearer S, Kambashi B, Godfrey-Faussett P. HIV-1 and recurrence, relapse, and reinfection of tuberculosis after cure: a cohort study in South African mineworkers. Lancet 2001; 358: 1687-1693.

12 Roche P, Bastian I, Krause V, et al. Tuberculosis notifications in Australia, 2005. Commun Dis Intell 2007; 31: 71-80.

13 Marks GB, Bai J, Simpson SE, Sullivan EA, Stewart GJ. Incidence of tuberculosis among a cohort of tuberculinpositive refugees in Australia: reappraising the estimates of risk. Am J Respir Crit Care Med 2000; 162: 1851-1854.

14 New South Wales Department of Health, Policy directive. Chemotherapy. www.health.nsw.gov.au/policies/PD/2005/ pdf/PD2005_071.pdf Date last updated: 25 January 2005. Date last accessed: 9 July 2008.

15 Kamerbeek J, Schouls L, Kolk A, et al. Simultaneous detection and strain differentiation of Mycobacterium tuberculosis for diagnosis and epidemiology. J Clin Microbiol 1997; 35: 907-914.

16 Supply P, Lesjean S, Savine E, Kremer K, van Soolingen D, Locht C. Automated high-throughput genotyping for study of global epidemiology of Mycobacterium tuberculosis based on mycobacterial interspersed repetitive units. J Clin Microbiol 2001; 39: 3563-3571.

17 van Embden JD, Cave MD, Crawford JT, et al. Strain identification of Mycobacterium tuberculosis by DNA fingerprinting: recommendations for a standardized methodology. J Clin Microbiol 1993; 31: 406-409.

18 Fleiss J. Statistical Methods for Rates and Proportions. 2nd Edn. New York, John Wiley \& Sons, 1981; pp. 14-15.

19 Dobler CC, Marks GB, Simpson SE, Crawford AB. Recurrence of tuberculosis at a Sydney chest clinic between 1994 and 2006: reactivation or reinfection? Med J Aust 2008; 188: 153-155.

20 Brudey K, Driscoll JR, Rigouts L, et al. Mycobacterium tuberculosis complex genetic diversity: mining the fourth international spoligotyping database (SpolDB4) for classification, population genetics and epidemiology. BMC Microbiol 2006; 6: 23.

21 Hong Kong Chest Service, British Medical Research Council. Controlled trial of 2, 4, and 6 months of pyrazinamide in 6-month, three-times-weekly regimens for smear-positive pulmonary tuberculosis, including an assessment of a combined preparation of isoniazid, rifampin, and pyrazinamide: results at 30 months. Am Rev Respir Dis 1991; 143: 700-706.

22 Mitchison DA. Role of individual drugs in the chemotherapy of tuberculosis. Int J Tuberc Lung Dis 2000; 4: 796-806. 
23 Cobelens FG, van Deutekom H, Draayer-Jansen IW, et al. Risk of infection with Mycobacterium tuberculosis in travellers to areas of high tuberculosis endemicity. Lancet 2000; 356: 461-465.

24 Ormerod LP, Green RM, Gray S. Are there still effects on Indian subcontinent ethnic tuberculosis of return visits?: a longitudinal study 1978-97. J Infect 2001; 43: 132-134.

25 World Health Organization, International Union Against Tuberculosis and Lung Disease, Royal Netherlands Tuberculosis Association. Revised international definitions in tuberculosis control. Int J Tuberc Lung Dis 2001; 5: 213-215.

26 Kamolratanakul P, Sawert H, Lertmaharit S, et al. Randomized controlled trial of directly observed treatment (DOT) for patients with pulmonary tuberculosis in Thailand. Trans R Soc Trop Med Hyg 1999; 93: 552-557.
27 Walley JD, Khan MA, Newell JN, Khan MH. Effectiveness of the direct observation component of DOTS for tuberculosis: a randomised controlled trial in Pakistan. Lancet 2001; 357: 664-669.

28 Jelfs P, Sintchenko V, Gilbert GL. Genotyping of Mycobacterium tuberculosis in New South Wales: results from 18 months of a statewide trial. NSW Public Health Bull 2006; 17: 81-85.

29 Merritt TD, Sintchenko V, Jelfs P, et al. An outbreak of pulmonary tuberculosis in young Australians. Med J Aust 2007; 186: 240-242.

30 Shamputa IC, Rigouts L, Eyongeta LA, et al. Genotypic and phenotypic heterogeneity among Mycobacterium tuberculosis isolates from pulmonary tuberculosis patients. J Clin Microbiol 2004; 42: 5528-5536. 